\title{
Component-based Development Process and Component Lifecycle
}

\author{
Ivica Crnkovic ${ }^{1}$, Stig Larsson ${ }^{2}$ and Michel Chaudron ${ }^{3}$ \\ ${ }^{1}$ Mälardalen University, Västerås, Sweden \\ ${ }^{2}$ ABB Corporate Research, Västerås, Sweden \\ ${ }^{3}$ Technical University Eindhoven, Eindhoven, The Netherlands
}

In recent years component-based development has become an established approach. Component-based Software Engineering (CBSE) that deals with the entire lifecycle of component-based products has been focused on technologies related to design and implementation of software components and systems built from software components. The experience has shown that pure technologies alone are not enough. A CBSE approach requires certain changes in development and life cycle processes. However, very few CBSE works, either research or practical, have addressed these topics. This paper describes principle differences of component-based and non- component based processes. Also we give an overview of a case study from a company that applies component-based approach.

Keywords: component-based software engineering, lifecycle processes.

\section{Introduction}

Component-based approach has in last years shown considerable success in many application domains. Distributed and web-based systems, desktop and graphical applications are typical examples of domains in which componentbased approach has been very successful. In these domains the general-purpose component technologies, such as COM, .NET, EJB, J2EE are used.

There is, however, very little development processes knowledge specific to component-based development.

This paper describes the characteristics of component-based processes, the reasons for this, and the differences from a non-component-based development process. From a case study it shows that component-based approach provides specific solutions in organization of a company.
The rest of the paper is organized as follows. Section 2 gives an overview of development processes. Section 3 discusses some basic characteristics of component-based approach and illustrates component-based activities in the "V" development process model. We illustrate a component-based development approach in an industrial case study in section 4. Finally, section 5 concludes the paper.

\section{Basic Characteristic of Lifecycle Process Models}

Lifecycle processes include all activities of a product or a system during its entire life, from the business idea for its development, through its usage and its completion of use. Different models have been proposed and exploited in software engineering, and different models have exhibited their (in)abilities to efficiently govern all activities required for a successful development and use of products. We can distinguish two main groups of models: Sequential and evolutionary. The sequential models define a sequence of activities in which one activity follows after a completion of the previous one. Evolutionary models allow performance of several activities in parallel without requirements on a stringent completion of one activity to be able to start with another one. Well known example of sequential models is waterfall model, or $\mathrm{V}$ model, and of evolutionary models, iterative and incremental development, or spiral model. 
Independently of the model type we can identify the basic activities present in any lifecycle process model. These activities are the following:

Requirements analysis and specification. The system's services, constraints and goals are established (i.e. a specification of what the system is supposed to do).

System and software design. An overall system and software architecture is established. A detailed design follows the overall design. Software design includes identifying and describing the fundamental software systems abstractions and their relationships.

Implementation and unit testing. The formalization of the design in an executable way, which can be composed of smaller units. Testing of the units follows their implementation.

System integration. The system units are integrated.

System verification and validation. Correctness of the complete system is verified and the system is validated with respect to the requirements.

Operation support and maintenance. A set of activates that are required for the expected performance of the system.

Disposal. A disposal activity, often forgotten in many lifecycle models, includes the phasingout of the system, i.e. a possible replacement by another system or a complete termination.

Not all models are suitable for all types of system lifecycles. Usually large systems, which include many stakeholders and whose development lasts a long period, prefer using sequential models. The systems which use new technologies are smaller and to which the time to market is important, usually explore evolutionary models that are more flexible and can show some results much earlier than sequential models. These models can be applied in a componentbased development, but require adaptation to the principles of component-based approach.

\section{Component-Based Lifecycle Process Models}

CBSE addresses challenges similar to those encountered elsewhere in software engineering.
Many of the methods, tools and principles of software engineering used in other types of system will be used in the same or similar way in CBSE. There is, however, one difference; CBSE specifically focuses on questions related to components and in that sense it distinguishes the process of "component development" from that of "system development with components".

\subsection{Building Systems from Components}

The main idea of the component-based approach is building systems from pre-existing components. This assumption has several consequences for the system lifecycle. First, the development processes of component-based systems are separated from development processes of the components; the components should already have been developed and possibly used in other products when the system development process starts. Second, a new separate process will appear: Finding and evaluating the components. Third, the activities in the processes will be different from the activities in non- componentbased approach; for the system development the emphasis will be on finding the proper components and verifying them, and for the component development, design for reuse will be the main concern.

There is a difference in requirements and business ideas in these two cases and different approaches are necessary. Components are built to be used and reused in many applications, some possibly not yet existing, in some possibly unforeseen way

System development with components is focused on the identification of reusable entities and relations between them, beginning from the system requirements and from the availability of already existing components [1][4]. Much implementation effort in system development will no longer be necessary, but the effort required in dealing with components: locating them, selecting those most appropriate, testing them, etc. will increase [6].

We do not only recognize different activities in the two processes, but also find that many of these activities can be performed independently 
of each other. In reality, the processes are already separate as many components are developed by third parties, independently of the system development. Even components being developed internally in an organization which uses these very same components, are often treated as separate entities developed separately.

Let us discuss these differences in more detail. Figure 1 shows a V development model adapted to component-based approach.

We use $\mathrm{V}$ model as this model is widely used in many organizations - typically large organization building complex long-life products, such as cars or robots. In this model the process starts in a usual way by requirements engineering and requirements specification, followed by system specification. In a non-component-based approach the process would continue with the unit design, implementation and test. Instead of performing this activities that often are time and efforts consuming, we simply select appropriate components and integrate them in the system. However, two problems appear here which break this simplicity: (i) It is not obvious that there is any component to select, and (ii) the selected component only partially fits our overall design. The first fact shows that we must have a process for finding components. This process includes activities for finding the components, and then the component evaluation. The second fact indicates for a need of component adaptation and testing before it can be integrated into the system. And of course there must be a process of component development, this being independent of the system development process.

Figure 1 also shows a simplified and idealized process. Its assumption is that the components selected and used are sufficiently close to the units identified in the design process, so that the adaptation process requires (significantly) less efforts than the units' implementation. Further, it does not consider what happens in the maintenance process; what happens if a system malfunctions due to a problem that has occurred in a component, or due to incompatibilities of the components. This indicates that the componentbased approach is not only limited to the development process, or part of the development process, but to the entire lifecycle. At a very early stage, in the Requirements and Design phases,

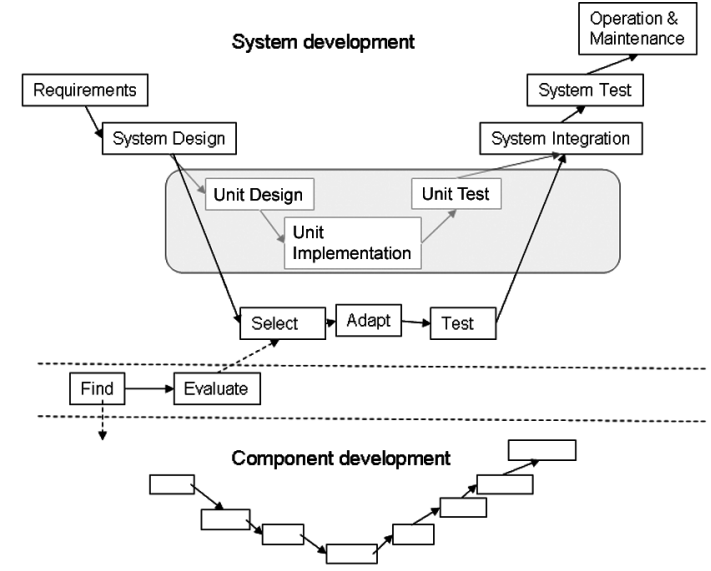

Fig. 1. V development process for CBD.

the system requirements engineers and system architects must be aware about the availability of already existing components.

A more realistic process is shown in Figure 2. Let us look at the activities in different phases of the development process in more detail.

Requirements analysis and specification. In this phase one important activity is to analyze the possibility of realizing the solutions that will meet these requirements. In a component-based approach this implies that it is necessary to analyze whether these requirements can be fulfilled with available components. This means that the requirements engineers must be aware of the components that can possibly be used. Since it is not likely that appropriate components can always be found, there is a risk that the new components have to be implemented. To keep with component-based approach (and utilize its advantages) one possibility is to negotiate the requirements and modify them to be able to use the existing components.

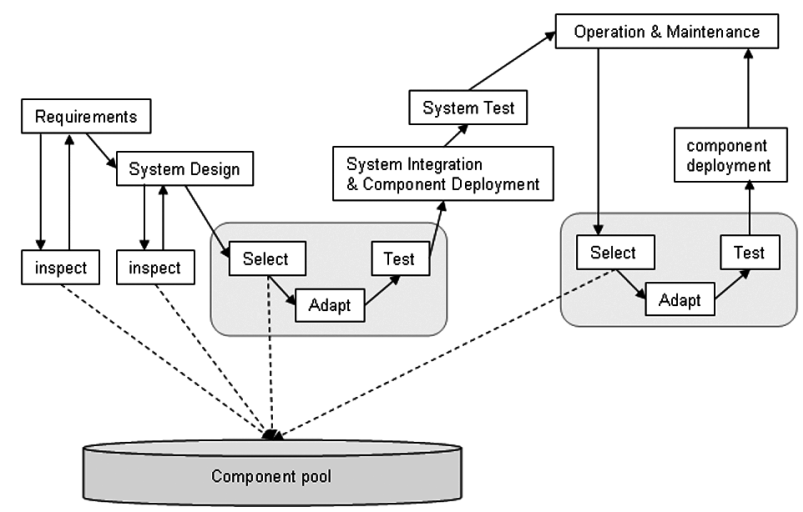

Fig. 2. A detailed V development process for CBD. 
System and software design. Similar to the requirements specification phase the system specification and design are strongly related to the availability of the components. The potential components are complying with a particular component model. One could assume that it would be possible to use components implemented in different component technologies, but in practice it is very difficult to achieve interoperability between different component models. Particular component model requires a particular architectural framework, and the application is supposed to use this framework. This has a direct impact on architectural decisions. For example, if the component model requires a client-server architecture style, it is obvious that the application will use that style and not another one (for example pipe-filter). This will put limitations on the system design. Also, other properties of components can have a direct influence on the design decisions. For this reason the design process is tightly connected to the availability of the components.

Implementation and unit testing. When building component-based system, an ideal case is to build an application by direct integration of components, i.e. connecting components directly. The "glue code" is a code that specifies this connection. In practice the role of the glue code will also include adaptation of the components, and even implementation of new functions. In an ideal case the components themselves are already built and tested. However, the component tests in isolation are not sufficient. Often, design units will be implemented as assemblies of several components and possibly a glue code. These assemblies must be tested separately since an assembly of correct components may be incorrect, although the components themselves are correct [3].

System Integration. The integration process includes integration of standard infrastructure components that build a component framework and the application components. The integration of a particular component into a system is called a component deployment. Unlike the entire system integration, a component deployment is a mechanism for integration of particular components - it includes download and registering of the component.

System verification and validation. The standard test and verification techniques are used here. A specific problem for component-based approach is location of error, especially when components are of "black box" type and delivered from different vendors. Typically, a component can exhibit an error, but the cause of the malfunction lies in another component. Contractual interfaces play an important role in checking the proper input and output from components. These interfaces enable a specification of input and output and checking the correctness of data.

Operation support and maintenance. The maintenance process includes some steps that are similar to the integration process: A new or modified component is deployed into the system. Also, it may be necessary to change the glue code. In most of the cases an existing component will be modified or a new version of the same component will be integrated into the system. Once again, new problems caused by incompatibility between components, or by broken dependencies, may occur. This means that the system must be verified (either formally, or by simulation, or by testing).

In comparison with a non-component-based approach, in a component-based development process there are significantly less efforts in programming, but the verification and testing require considerably more efforts. The verification activity is repeated in several phases, with slightly different goals:

- Verifying component in isolation;

- Verifying components in an assembly;

- Verifying the system when a component has been deployed into the system.

\subsection{Building Reusable Components}

The process of building components can follow an arbitrary development process model. However, any model will require certain modification to achieve the goals; in addition to the demands on the component functionality, a component is built to be reused. Reusability implies generality and flexibility, and these requirements may significantly change the component characteristics. For example there might be a requirement for portability, and this requirement could imply a specific imple- mentation solution (like choice of programming language, implementation of an intermediate level of services, programming style, etc.). The generality requirements imply often more functionality and require more design and development 
efforts and more qualified developers. The component development will require more efforts in testing and specification of the components. The components should be tested in isolation, but also in different configurations. Finally, the documentation and delivery will require more efforts since the extended documentation is very important for increasing understanding of the component. An example of extended component specification can be found in ROBOCOP component model [5]; a component is specified by a row of modules: executable model, functional model, simulation model, resource model, etc. Each model includes a corresponding documentation.

\section{Industrial Case of Component-Based Process Model}

We give here a short overview of a case study: a process model used in a large international consumer electronics company. The case study was performed by four researchers in intensive interviews with different stakeholders of the development projects: system architects, component architects, developers, project leaders, the management, the quality assurance and test people, and principal specialists.

The development divisions of the company are placed in four different countries and they produce numerous products with different variants and models. The company has adopted component-based development using productline architecture. The component model is internally developed and most of the tools are internally developed. The reason for that are specific requirements of the domain: low resource usage, high availability, and soft real-time requirements.

The component model follows the basic principles of CBSE: The components are specified by interfaces which distinguish "require" from "provide" interfaces. In addition to functional specification, the interface includes additional information; interaction protocols, timeliness properties, and the memory usage. The component model enables a smooth evolution of the components as it allows existence of multiple interfaces. The model has a specific characteristic; it allows hierarchical compositions: a composite component is treated as a standard component and it can be further integrated in another component. The components are also developed internally, but their development is separated from the development of the products.

The product-line architecture identifies the basic architectural framework. The product architecture is shown in Figure 3.

The product architecture is a layered architecture which includes (i) operating system, (ii) the component framework which is an intermediate level between domain-specific services and operating, (iii) core components which are included in all product variants, and (iv) application components that are usually different for different product variants.

Complementary to this horizontal layering there is a vertical structuring in form of subsystems. Subsystems are also related to organizational structures; they are responsible for development and maintenance of particular components. The overall process is designed as shown in Figure 4.

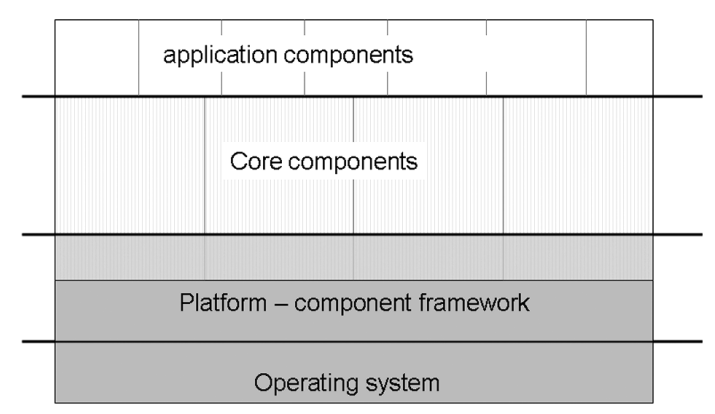

Fig. 3. Product software architecture.

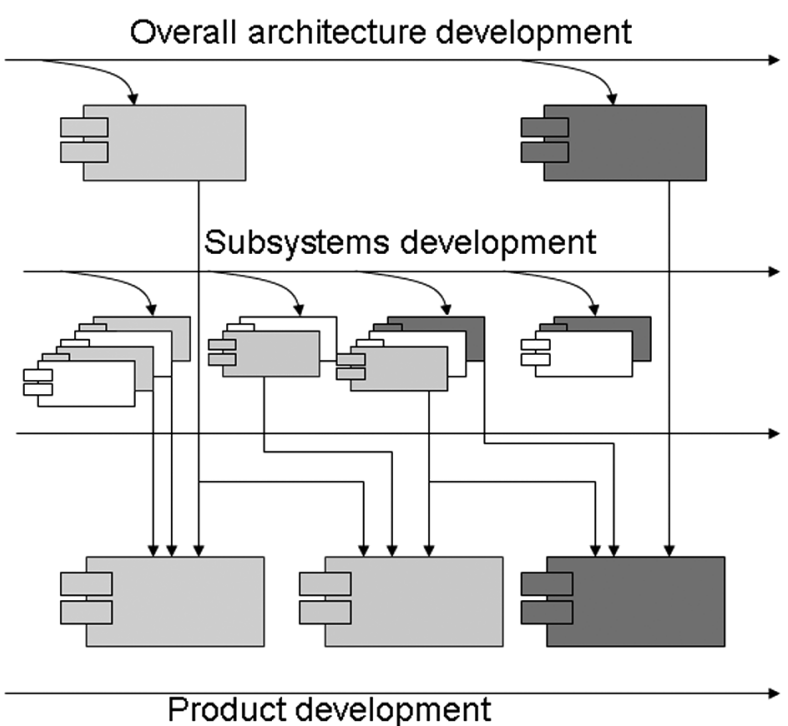

Fig. 4. Overall development process. 
In the overall process there are three sets of independent parallel processes: (i) An overall architecture and platform development process are responsible for delivering new platforms and basic components, (ii) subsystem development processes which deliver a set of components that provide different services, and (iii) the product development process which is basically an integration process. This process arrangement makes it possible to deliver new products every six months, while the development of subsystem components takes typically between 12 and 18 months. The specific feature of these projects is that all deliverables have the same form. A deliverable is a software package defined as a component. Two main documents belong to every deliverable: component interface specification and component sheet; the first document describing the interconnection, the second describing the component internals.

Although the overall development and production is successful, the process suffers from several drawbacks. The most serious is late discovery of errors, due to interface or architectural mismatches, insufficient specifications of semantics of the components, or due to inappropriate interfaces. Also, the problems related to encapsulation of a service in components often occur; due to functional overlaps, or some requirements affecting the architecture, it is difficult to decide in which components a particular function will be implemented. All these problems indicate that it is difficult to perform the processes independently; negotiation between different subsystems and agreement in many technical details between different teams are necessary. For this reason the processes are not completely separated. They are distributed among several projects and there is an overall project that coordinates them all.

The processes have a strong support in the project and organization structure (see Figure $5)$.

The system architect and the management have overall responsibilities for requirements, policies, product line architecture, products visions and long term goals. The project architect has a responsibility for the overall project which results in a line of products. He/she coordinates the architectural design of the product family and subsystems. The test and quality-assurance

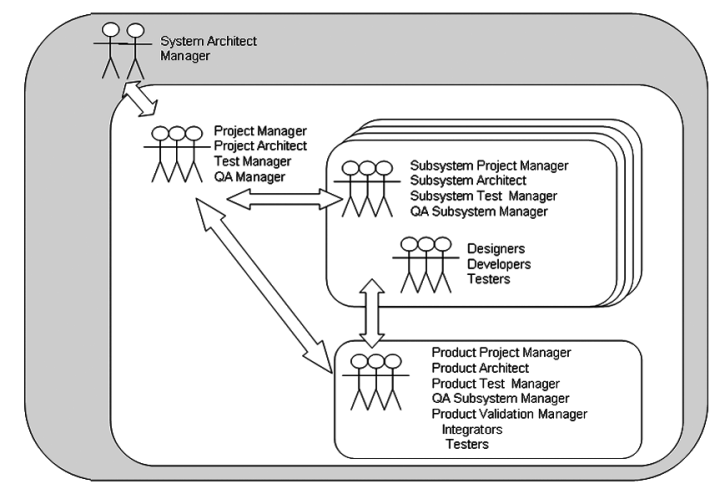

Fig. 5. Project organization structure.

(QA) managers have similar role in their domains: to ensure coordination and compatibility of tests and quality processes. Subsystem architects provide the designs of their subsystems and coordinate the design decisions with other subsystems. Each subsystem has a test team and a QA manager whose responsibility is the quality of delivered subsystem components. The integration team which works in delivery projects is represented by a product architect, QA and test managers who coordinate the activities with other projects. We can observe that project teams have many "nonproductive" stakeholders. This is in line with the component-based approach - more efforts must be put on overall architecture and testing, and less on the implementation itself. Development processes in our case are manly of an evolutionary model. The platform, the subsystems and the products are developed in several iterations until the desired functionality and quality are achieved. This requires synchronizations of iterations.

\section{Conclusion}

A component-based approach cannot be fully utilized if development processes and even development organizations are not adopted according to basic principles of CBSE. Since this approach aims for increased reusability of existing components, the efforts for implementations decrease, and the efforts for system verification increase. This requires adjustments of the development processes. 
By an industrial case study we have pointed out the difficulties to achieve a complete separation of the development processes of systems from the components, as well as the need for a project organization which puts more emphasis on architectural issues and on system and components verification.

\section{Acknowledgments}

The authors would like to thank Chritiene Aarts for his enormous help in organizing the interviews and all the interviewees who took their valuable time for the interviews.

\section{References}

[1] L. BASS, P. Clements AND R. KAZMAN, Software Architecture in Practice, Addison Wesley, 1998.

[2] V. BorghofF, R. PARESI, (editors), Information Technology for Knowledge Management, New York: Springer Verlag; 1998.

[3] IVICA CRNKOVIC AND MAGNUS LARSSON (editors), Building Reliable Component-Based Software Systems, Artech House Publishers, ISBN 1-58053-3272, 2003.

[4] D. Garlan, R. Allen AND J. OCKerbloOM, Architectural Mismatch: Why Reuse is so Hard, IEEE Software, Vo. 12, issue 6, 1995.

[5] ITEA project, ROBOCOP-Robust Open Component Based Software Architecture for Configurable Devices Project http://www.hitechprojects.com/euprojects/robocop.

[6] M. Morisio, C. B. Seaman, A. T. Parra, V.R. BASIL, S.E. KRAFT AND S.E. CONDON, Investigating and Improving a COTS-Based Software Development Process, In Proceedings, 22nd ICSE, ACM Press, 2000.
Recived: June, 2005. Accepted: October, 2005

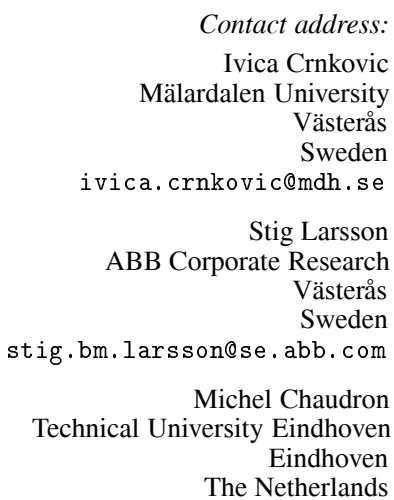

IVICA CRNKOVIC is a professor of industrial software engineering at Mälardalen University where he is the administrative leader of the software engineering laboratory and the scientific leader of the industrial software engineering research. His research interests include component-based software engineering, software configuration management, software development environments and tools, as well as software engineering in general. Professor Crnkovic received an M.Sc. in electrical engineering in 1979, an M.Sc. in theoretical physics in 1984, and a Ph.D. in computer science in 1991, all from the University of Zagreb, Croatia.

Michel R. V. CHAUDRON is an assistant professor at the Eindhoven University of Technology and a researcher in the System Architecture and Networking group. His research interests include software architecture, empirical software engineering and component-based software engineering. He received his MSc and his $\mathrm{PhD}$ from the Universiteit Leiden and worked as a consultant in traffic and transport telematics.

STIG LARSSON is responsible for the product development process improvement initiative at $\mathrm{ABB}$. He has occupied different development and management positions in $\mathrm{ABB}$ for the last 20 years. His professional interest is in product development processes and software architecture. $\mathrm{He}$ received his MSc in electrical engineering from the Royal Institute of Techonoloy in Stockholm. 